\title{
New Processing and Characterization Approaches for Achieving Full Performance of High Temperature Superconducting Tapes of $(\mathrm{Bi}, \mathrm{Pb})_{2} \mathrm{Sr}_{2} \mathrm{Ca}_{2} \mathrm{Cu}_{3} \mathrm{O}_{\mathrm{x}}$.
}

\author{
Eric E. Hellstrom and David C. Larbalestier, University of Wisconsin-Madison
}

\section{EXECUTIVE SUMMARY}

The thrust of this research was to identify and understand current limiting mechanisms (CLMs) that limit the current carrying capacity of $\left(\mathrm{Bi}, \mathrm{Pb}_{2} \mathrm{Sr}_{2} \mathrm{Ca}_{2} \mathrm{Cu}_{3} \mathrm{O}_{\mathrm{x}}\right.$ (2223) in Agsheathed wire. Our program concentrated on developing new methods to identify CLMs at the micrometer scale and new processing techniques to eliminate CLMs. All of the DOE Superconductivity Partnership Initiative (SPI) programs are using 2223 wire, so increasing the critical current density $\left(\mathrm{J}_{\mathrm{c}}\right)$ in the wire can improve the technical performance of the demonstration projects, and at the same time it can decrease the cost of the wire. The important cost metric for superconducting wire is $\$ / \mathrm{kA} \cdot \mathrm{m}$, so increasing $\mathrm{J}_{\mathrm{c}}$, which is in the denominator, decreases the wire cost.

The obvious CLMs were micrometer size obstacles in the 2223 ceramic that block current flow, including: misaligned grains, cracks, pores, and nonsuperconducting phases. Pores and cracks - regions where there is no superconductor or the grains are not physically connected to one another - cannot carry supercurrent, so they were the first CLMs we tried to eliminate with improved processing. Prior to the contract, we had started investigating overpressure (OP) processing with Williams at ORNL to heal cracks and remove pores. OP processing, which is a variant of hot isostatic pressing (HIP), uses an $\mathrm{Ar} / \mathrm{O}_{2}$ gas mixture to apply a high pressure (up to $200 \mathrm{~atm}$ ) to compress the sample and to set the oxygen partial pressure $\left(\mathrm{pO}_{2}\right)$ to form 2223. Williams had a static pressure system we used to demonstrate that OP processing healed cracks and densified the wire, but the static system limited the processing parameters we could investigate. We proposed building a new gas-flow OP system to expand the experimental capabilities and to investigate new processing routes using the gas-flow OP system. Using the gas-flow OP system, we established new world records in 2003 for $\mathrm{J}_{\mathrm{C}}$ and $\mathrm{I}_{\mathrm{c}}$. These records were finally matched by Sumitomo Electric Company in early 2006.

The finest scale at which we could probe the local electromagnetic properties of a sample was about $100 \mu \mathrm{m}$ at the beginning of the contract. This was done by attaching voltage taps (10 $\mu \mathrm{m}$ diameter wires) about $100 \mu \mathrm{m}$ apart on the 2223 conductor, and measuring the local I-V characteristics between each set of voltage taps. However, the largest CLMs were 2-3 times smaller than this length scale, and most CLMs were even much smaller. The original proposal was to investigate new methods to identify specific regions in samples that contained CLMs from their electromagnetic response, then to examine these regions of the sample using microstructural techniques to identify the 
CLM. We extended the use of magneto-optic (MO) imaging and magneto-optic current reconstruction (MOCR) and began developing a low-temperature laser scanning microscope (LTLSM) to show local current flow and local current dissipation, respectively, with a resolution of $\sim 5 \mu \mathrm{m}$. With MOCR we were able to show that local $\mathrm{J}_{\mathrm{c}}$ in small regions of OP processed 2223 wire was as high as $300 \mathrm{kA} / \mathrm{cm}^{2}$ at $77 \mathrm{~K}$, which was 5-6 times higher than the average $\mathrm{J}_{\mathrm{c}}$ measured across the whole sample.

\section{SUMMARY OF RESEARCH ACCOMPLISHMENTS}

This research involved two thrust areas: (1) Overpressure and other innovative processing methods and (2) Characterization at small length scales.

The proposed work for each of these thrust areas is listed below along with the accomplishments for each thrust area.

Overpressure and other innovative processing methods - [Work carried out by Eric Hellstrom and David Larbalestier, Yongwen Yuan (PhD student), Jianyi Jiang (post doc then assistant scientist))

\section{Proposed work}

- Build a flow-type overpressure system.

- Establish freeze dry capability to synthesize Bi-2223 precursor powder for tapes and to react into pure $\mathrm{Bi}-2223$ powder.

- Carry out slide heat treatment processing in the overpressure system.

- Do overpressure processing using pre-reacted Bi-2223 powder.

- Examine effect of overpressure processing on Bi-2223 with different conductor geometries.

- Innovate and refine the process and evaluate ways to reduce the pressure-timetemperature profile.

The overpressure (OP) processing research developed a basic understanding how this processing method could eliminate pores and cracks as current limiting mechanisms (CLM) in 2223. We extended our understanding of processing methods by carrying out through-processing quench studies that allowed us correlate the development of the microstructure during with the corresponding electromagnetic properties. This was the first study of its kind on a high-temperature superconductor.

Overpressure Processing

In conjunction with Williams at ORNL, Yuan designed and built a gas-flow OP system. It consists of an externally-heated Inconel 617 tube with a uniform hot zone 2.5 $\mathrm{cm}$ in diameter and $\sim 5 \mathrm{~cm}$ long. The maximum pressure is $200 \mathrm{~atm}$ at the maximum working temperature of $900^{\circ} \mathrm{C}$. It uses high-pressure (400 atm) premixed $\mathrm{Ar} / \mathrm{O}_{2}$ gas to set the desired $\mathrm{pO}_{2}(\sim 0.075 \mathrm{~atm})$ at the desired total pressure, which in these studies was 
$\sim 150$ atm. The gas flow rate through the OP system was controlled with a gas regulator on the down-stream side of the system. The design has proved to be very robust.

Since we only had one OP system and many $1 \mathrm{~atm}$ furnaces, we developed processing schemes at $1 \mathrm{~atm}$, and then carried out the best $1 \mathrm{~atm}$ heating schedules in the OP system. Using $1 \mathrm{~atm}$ studies, Jiang developed a multi-step processing schedule for the final heat treatment of the 2223 wire that led to high $\mathrm{J}_{\mathrm{c}}$. A key finding from the study was that $\mathrm{J}_{\mathrm{C}}$ increased by adding a post annealing step after the second heat treatment. The post annealing consisted of cooling the sample to $<\sim 500^{\circ} \mathrm{C}$ after the second heat treatment, then heating it back to $\sim 780^{\circ} \mathrm{C}$ for $\sim 24 \mathrm{~h}$, then cooling to room temperature.

Our studies showed that OP processing is most effective when a precursor material is converted into 2223 under pressure. This heals cracks and removes porosity, which can increase $\mathrm{J}_{\mathrm{c}}$. We found that when we began with pure 2223 powder, OP processing was unable to completely densify the sample. We could only get densification by going to a high enough temperature to partially melt the 2223, which converted back to 2223 on cooling. However, this did not yield high $\mathrm{J}_{\mathrm{c}}$. In fact, we found that all the OP samples that began with pure 2223 had low $\mathrm{J}_{\mathrm{c}}$. We initially thought that OP processing would simplify 2223 processing decreasing it from two heat treatments to a single heat treatment, plus eliminating the intermediate rolling. However, our studies showed that we achieved higher $\mathrm{J}_{\mathrm{c}}$ using a two-step heat treatment where the first heat treatment was done at $1 \mathrm{~atm}$ pressure, and OP processing was used for all heat treatments after the intermediate roll. Our studies showed that the intermediate rolling between the first and second heat treatment is important because it crushes the nonsuperconducting phases in the wire (as well as some of the 2223 grains, which causes the cracks), mixes the phases, and presses the phases together. These are the same steps one uses in solid-state synthesis when grinding a pellet then pressing the resulting powder into a pellet.

Using OP processing we achieved a record $\mathrm{J}_{\mathrm{c}}$ value of $30.2 \mathrm{kA} / \mathrm{cm}^{2}$ at $0.1 \mathrm{~T}, 77 \mathrm{~K}$, and in another wire, with slightly different dimensions, we achieved $202 \mathrm{~A}$ in self field at $77 \mathrm{~K}$. Figure 1 shows the microstructure of samples produced with $1 \mathrm{~atm}$ and OP processing (148 atm). It shows that that OP processing eliminates pores and cracks. Close examination of the microstructure of OP samples show that OP processing has not eliminated all the pores and cracks, there are fewer smaller cracks and pores in the OP sample than the $1 \mathrm{~atm}$ sample. In addition, the OP sample still contains grains of nonsuperconducting phases that are black. The good surface finish after polishing the highdensity OP sample highlights the light gray streaks between the darker 2223 grains. These are 2212 grains that bridge 2223 grains in 2223 colonies.

Other innovative processing methods - Through-process studies of the microstructure and electromagnetic properties of samples quenched during post annealing.

Jiang showed that adding a post annealing step after the second heat treatment could increase $\mathrm{J}_{\mathrm{c}}$. We use the term "post anneal" in the broadest sense to designate any thermal process, other than continuous cooling to room temperature, after the second heat treatment (HT2). Specifically, they found that after HT2, if they rapidly cooled the 
sample from to room temperature, then reheated to $\sim 780^{\circ} \mathrm{C}, \mathrm{J}_{\mathrm{C}}$ increased by about $25 \%$. The microstructure after post annealing at $\sim 780^{\circ} \mathrm{C}$ showed $(\mathrm{Bi}, \mathrm{Pb})_{3} \mathrm{Sr}_{2} \mathrm{Ca}_{2} \mathrm{CuO}_{\mathrm{x}}(3221)$, which is a $\mathrm{Pb}$-rich phase. If the sample were reheated above $\sim 805^{\circ} \mathrm{C}$ then rapidly cooled, $\mathrm{J}_{\mathrm{C}}$ decreased to its original value and the 3221 phase had disappeared.

In his PhD studies, Yuan did through-processing quenching studies to investigate how the microstructure and resulting electromagnetic properties developed during post annealing. He quenched wire samples into oil at specified points in the post annealing heat treatment. We used MO imaging to show that the ceramic core had not cracked during the quench, so as to assure ourselves that our measured whole-sample $\mathrm{I}_{\mathrm{c}}$ values were not degraded in the quenched wires. Figure 2 shows a quench schedule, and Fig. 3 shows $I_{c}$ in samples quenched a various temperatures. Overall, $I_{c}$ more than doubled during the post anneal. It is striking that $\mathrm{I}_{\mathrm{c}}$ (self-field, $77 \mathrm{~K}$ ) tracks with the quench temperature during post annealing, increasing with decreasing quench temperature, and decreasing with increasing quench temperature. This large, rapid change in $\mathrm{I}_{\mathrm{c}}$ during the very last stages of the processing was a completely new and unexpected result. These through-process quench studies showed that the 3221 phase forms from liquid present at the end of HT2, and the SQUID 2212 index, which we developed in this study, shows that the amount of 2212 also generally increased during cooling and decreased during heating in the last stages of the heat treatment. The Kramer field $\left(\mathrm{H}_{\mathrm{K}}\right)$, which is one measure of flux pinning, also varied with the quench temperature, increasing with decreasing temperature and decreasing with increasing temperature.

1 Bar, SEM

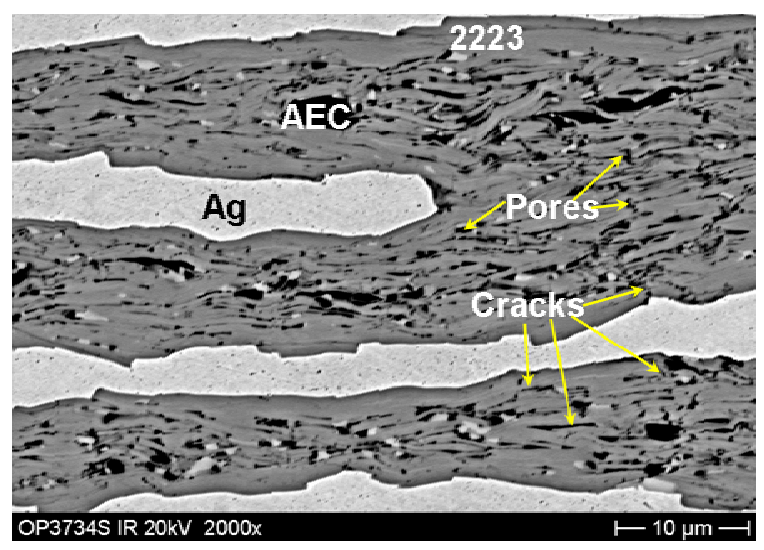

OP, SEM

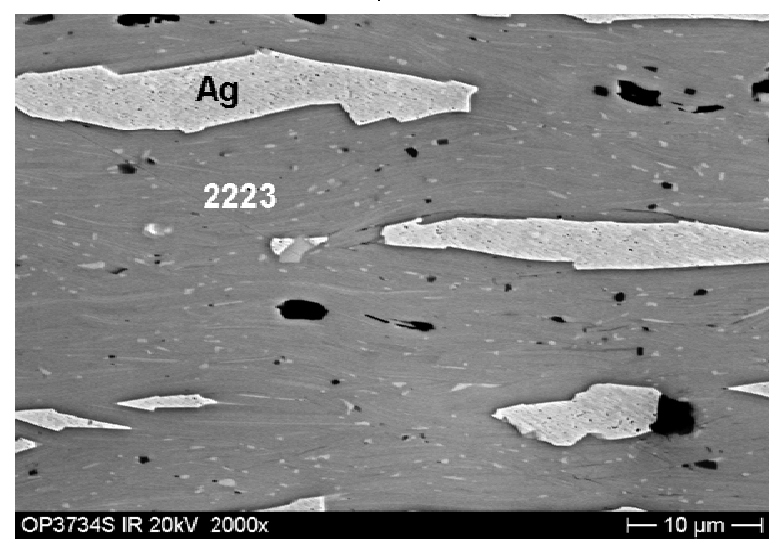

Figure 1 SEM images of the microstructure of fully-processed 2223 wire processed under (a) normal $1 \mathrm{~atm}$ conditions and (b) with OP processing with a total pressure of $148 \mathrm{~atm}$. In (a) the black regions are pores, cracks, and non-superconducting phases. In (b) the black regions are mainly non-superconducting phases. The small white spots in both images are grains of 3221 and the light gray streaks in (b) are grains of 2212. 


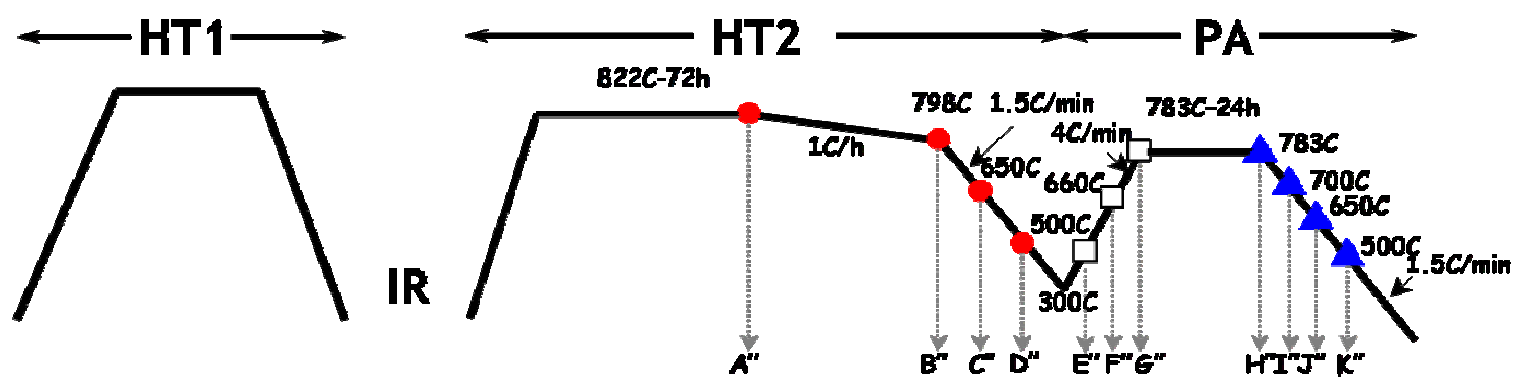

Figure 2. Quenching schedule for heat treatment 2 (HT2) used in these studies.

The quench studies showed that while many tens of hours, up to hundreds of hours, are needed to convert the precursor phases into 2223, the electromagnetic properties of fully-heat treated 2223 wire develop in the final few hours as the wire cools to room temperature. Further, our studies show that the increase in $\mathrm{I}_{\mathrm{C}}$ during cooling is mainly due to increases in flux pinning, which we believe is related to oxygen pick-up on cooling.

\section{Characterization at small length scales [Work carried out by Eric Hellstrom and David Larbalestier, Sandy Liao, (PhD student), Xueyan Cai (scientist), and Satyabrata Patnaik and Dima Abraimov (post docs))}

The ultimate goal of the local electromagnetic characterization was to develop methods identify regions of the sample that contain CLMs at the 1$5 \mu \mathrm{m}$ size scale. Once these regions are identified, other techniques such as SEM and TEM would be used to determine what the CLMs actually are. There were two major thrusts in our local electromagnetic characterization:

(1) magneto-optic (MO) characterization with magnetooptic current reconstruction (MOCR), and (2) low-temperature laser scanning microscopy (LTLSM).

\section{Proposed work}

- Investigate lithographic methods for making 10-50 $\mu \mathrm{m}$ spaced voltage taps and attempt to adapt these to

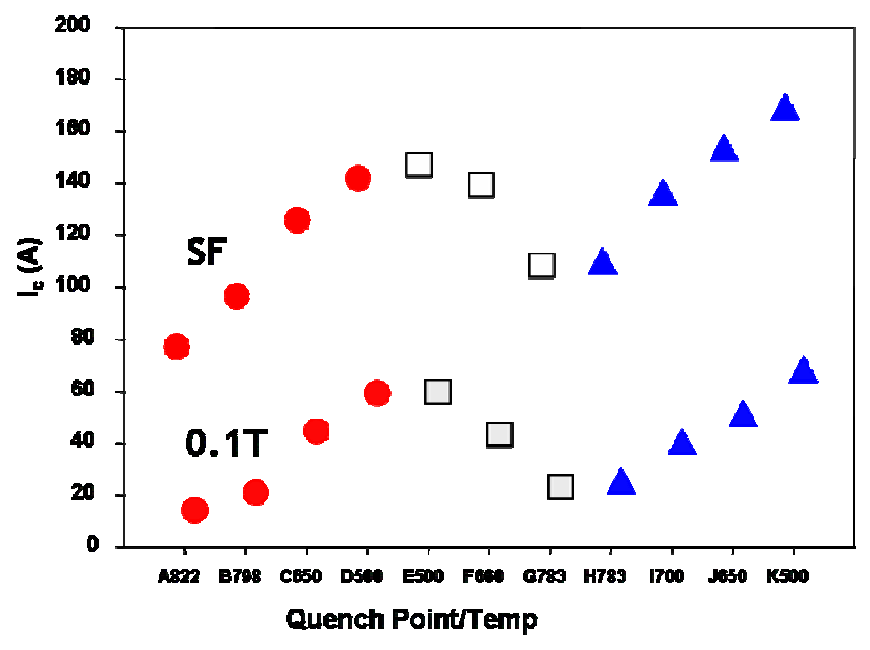

Figure 3. $\mathrm{I}_{\mathrm{c}}(77 \mathrm{~K})$ measured at self field (SF) and $0.1 \mathrm{~T}$ for samples quenched at different temperatures. The letters/numbers on the $\mathrm{x}$ axis correspond to the points in Fig. 2, and the numbers correspond to the quench temperature. $I_{c}$ increases with decreasing quench temperature and decreases with increasing quench temperature. We believe that the dominant reason for this change is that 2223 picks up oxygen during cooling, raising $\mathrm{I}_{\mathrm{c}}, \mathrm{T}_{\mathrm{c}}$ and $\mathrm{H}_{\mathrm{K}}$. 
cryogenic critical current measurements. Compare such methods to magneto optical current reconstructions.

- Perform quantitative magneto optical reconstructions of the local current density in our overpressure processed samples.

- Determine best means of measuring local current density on scales of 10-100 $\mu \mathrm{m}$ in a manner that supports the processing program.

- Apply chosen method(s) (local I-V, MO reconstructions, or high sensitivity magnetometry) to determine the location of specific current-limiting defects in filaments, and incorporate cross-sectional SEM studies to determine what these defects are.

\section{Magneto-optic imaging}

MO imaging shows magnetic penetration into a sample. The magnitude of $\mathrm{J}_{\mathrm{c}}$ at each point in the sample can be calculated from the MO image. This process is called magneto-optic current reconstruction (MOCR). To correlate the MO and MOCR images with the SEM image of the sample's microstructure requires excellent registry between these images. In her PhD studies, Liao cut trenches in her samples using the focused-ionbeam (FIB) SEM to create registry marks that were visible in the SEM images and in the MO images. Using this technique she achieved better than $1 \mu \mathrm{m}$ registry between the SEM images and the MO and MOCR images. This registry is better than the inherent resolution of MO and MOCR, which is between 1-5 $\mu \mathrm{m}$.

MOCR requires a slab geometry, so monocore wires were used for these studies. The MOCR images in Fig. 4 show a significant difference between samples processed in $1 \mathrm{~atm}$ and OP processed ( 150 atm). In the $1 \mathrm{~atm}$ sample, the high $\mathrm{J}_{\mathrm{c}}$ regions are mainly located at the edges of the 2223 core, whereas in the OP sample, the high $\mathrm{J}_{\mathrm{c}}$ regions are more uniformly distributed throughout the 2223 core. In addition, both samples show local regions whose $\mathrm{J}_{\mathrm{c}}$ is higher than the whole-sample $\mathrm{J}_{\mathrm{c}}$. However, in the OP sample, there are more local regions with high $\mathrm{J}_{\mathrm{c}}$, these are more uniformly distributed across the 2223 core, and the maximum $\mathrm{J}_{\mathrm{C}}$ is $\sim 300 \mathrm{kA} / \mathrm{cm}^{2}$, compared to $\sim 200 \mathrm{kA} / \mathrm{cm}^{2}$ in the $1 \mathrm{~atm}$ sample. When the maximum $\mathrm{J}_{\mathrm{c}}$ is limited to $50 \mathrm{kA} / \mathrm{cm}^{2}$ (Figs. $4 \mathrm{c}$ and $\mathrm{d}$ ), the MOCR image shows where the "average" and higher current flow in the sample. In the $1 \mathrm{~atm}$ sample, these regions are located mainly in the outer portions of the 2223 core and are not well connected. In contrast, in the OP sample they are spread across the entire cross section, and they are more uniform and continuous than in the $1 \mathrm{~atm}$ sample. This indicates better connectivity in the OP sample than in the 1 atm sample.

Figure 5 shows a combined MOCR and high-resolution SEM images of a monocore 2223 wire. Figure 5a shows the MOCR image superimposed over a low magnification SEM image of the sample. A high and low $\mathrm{J}_{\mathrm{c}}$ region indicated in Fig. 5a were imaged at higher magnification in Fig. 5b. Figures $5 c$ and $d$ show higher magnification images of the high and low $\mathrm{J}_{\mathrm{c}}$ regions. These images in Fig. $5 \mathrm{c}$ and $\mathrm{d}$ are at first glance quite similar. However, close inspection shows subtle differences in the 

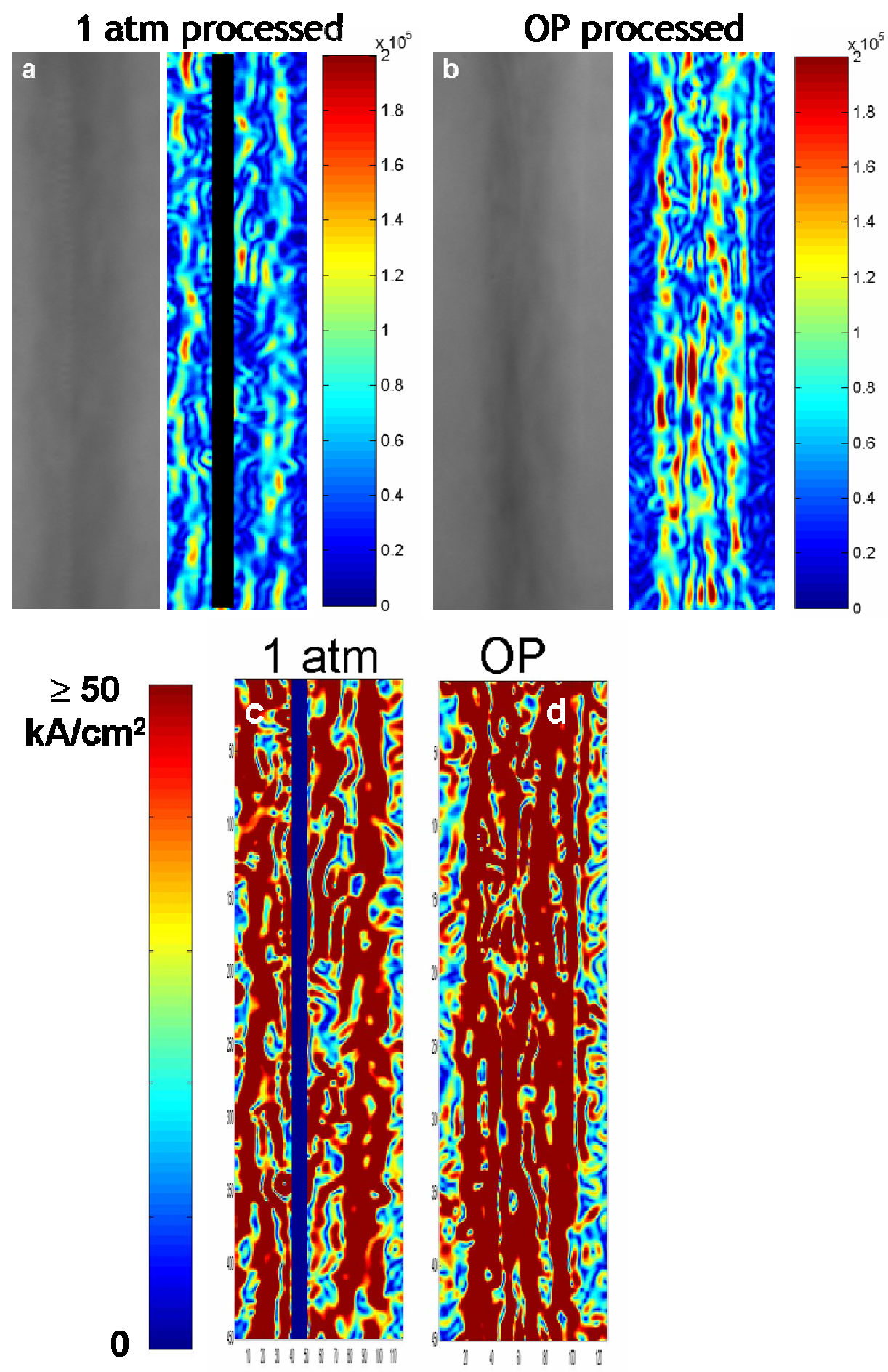

Figure 4. SEM and MOCR images from monocore wire with 1 atm (a and c) and OP processing (148 atm, b and d). The OP wire (a) has more local regions with the highest $\mathrm{J}_{\mathrm{c}}$ compared to the $1 \mathrm{~atm}$ wire (a), and these high $\mathrm{J}_{\mathrm{c}}$ regions are more uniformly distributed across the cross section in the OP sample. The MOCR images in $\mathrm{c}$ and $\mathrm{d}$ show all regions with $\mathrm{J}_{\mathrm{C}}>50 \mathrm{kA} / \mathrm{cm}^{2}$ together. These images show that in the OP sample (d) there are more local regions with high $\mathrm{J}_{\mathrm{c}}(>50$ $\mathrm{kA} / \mathrm{cm}^{2}$ ), they are better connected, and they are more uniformly distributed across the wire. 


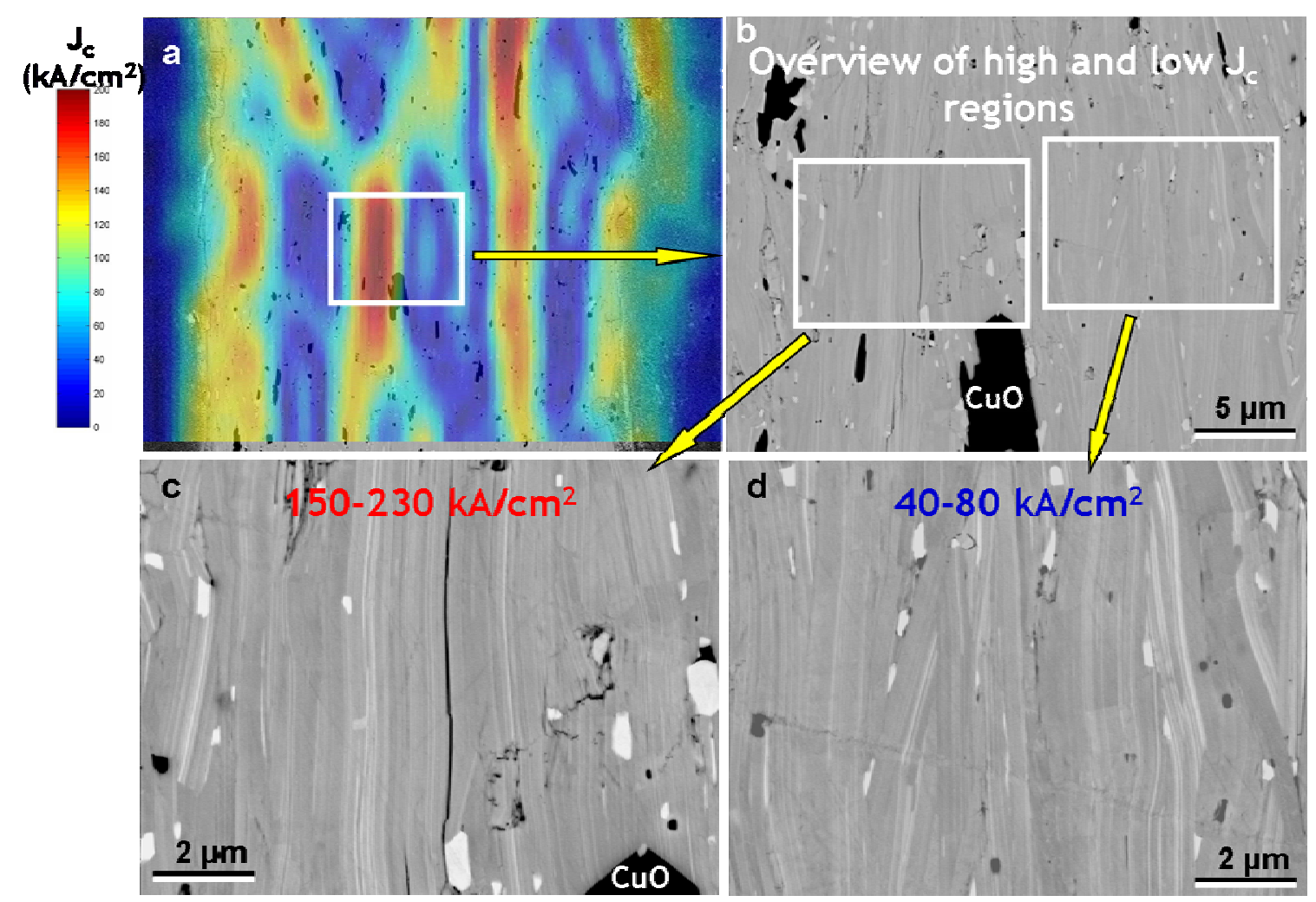

Figure 5 MOCR and SEM images for a monocore 2223 wire. The image in (a) has the MOCR image overlaid on the SEM image. The boxed region in (a) is shown in a higher magnification SEM image in (b). The high and low $\mathrm{J}_{\mathrm{c}}$ regions from (a) are boxed in (b) and shown at higher magnification in (c) and (d). The grains are better aligned along the wire axis in the high $\mathrm{J}_{\mathrm{c}}$ region (c) and less well aligned in the low $\mathrm{J}_{\mathrm{C}}$ region in (d).

microstructures. The slab geometry used for the MOCR has the $2223 \mathrm{ab}$ planes running vertically and into and out of the paper. The $2223 \mathrm{c}$ axis is horizontal. Current would flow through the sample in the vertical (= North-South (N-S)) direction. In the high- $\mathrm{J}_{\mathrm{c}}$ region (Fig. 5c), the ab planes are almost all aligned vertically, which allows the current to easily transfer from one grain to the next in the $\mathrm{a}$ and $\mathrm{b}$ directions. The current can flow relatively unobstructed in the N-S direction. There are cracks between the 2223 grains, but because these run vertically, they do not block current flow in the N-S direction.

In the low- $\mathrm{J}_{\mathrm{C}}$ region, the 2223 grains are generally aligned in the vertical direction, but not nearly as well aligned as in the high $\mathrm{J}_{\mathrm{c}}$ region. Current that flows in the N-S direction requires a component of c-axis current transport to move from one misaligned grain to the next, which decreases $\mathrm{J}_{\mathrm{c}}$. We identified such regions for later TEM investigation by our collaborator Terry Holesinger (LANL). The plans were to cut out regions of interest by FIB, but the contract ended before this could be completed.

MOCR was done on monocore tape that was oil-quenched at temperatures similar to those in Fig. 2 after the first heat treatment and after HT2. Figure 6 is a plot of the 

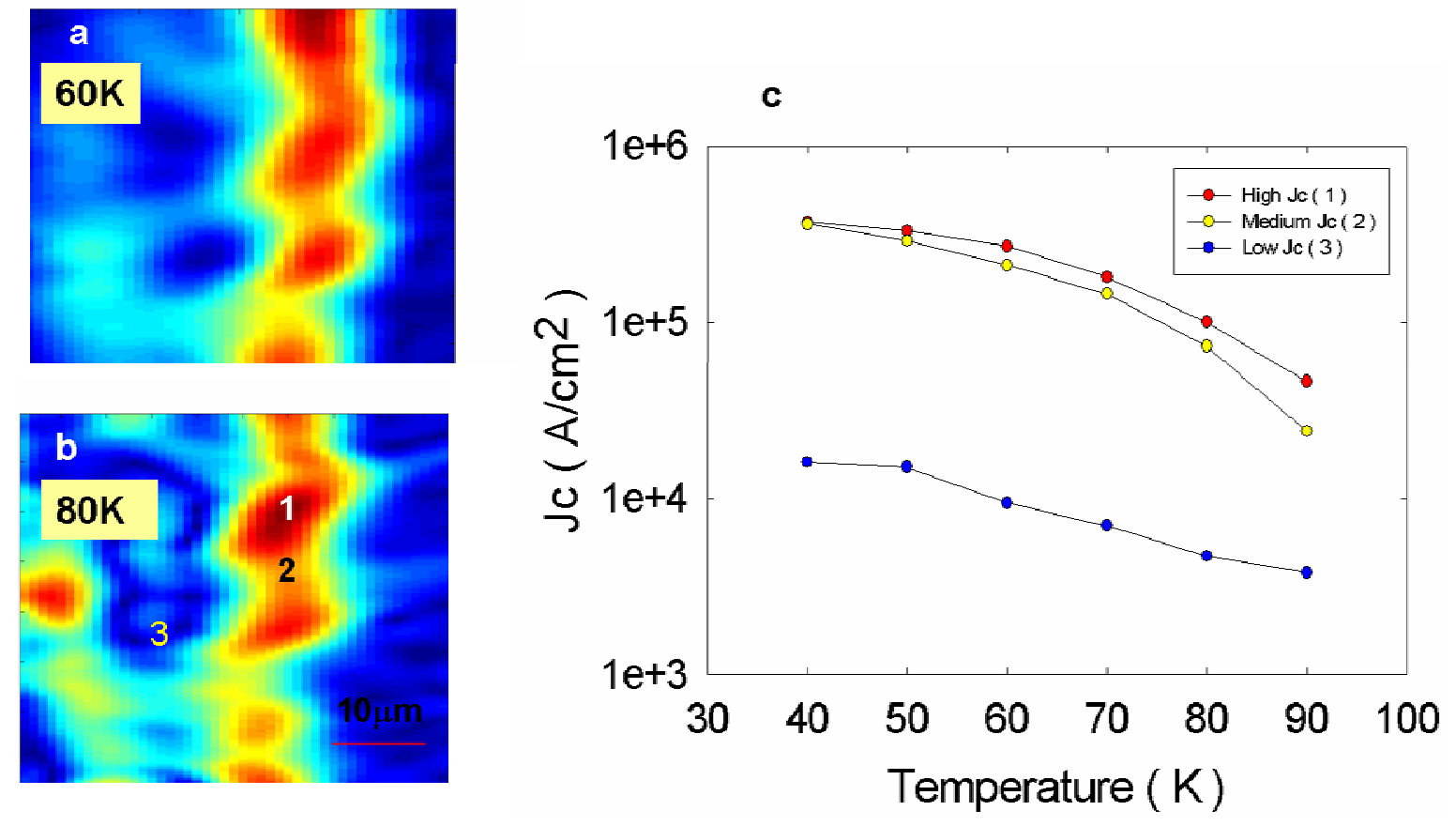

Figure 6 MOCR images of a sample at (a) $60 \mathrm{~K}$ and (b) $80 \mathrm{~K}$. $\mathrm{J}_{\mathrm{C}}$ in a (1) high, (2) medium, and (3) low $\mathrm{J}_{\mathrm{c}}$ region is plotted as a function of temperature in (c). There is a smooth change in $\mathrm{J}_{\mathrm{c}}$ with temperature in all three regions.

average local $\mathrm{J}_{\mathrm{c}}$ of the sample (at $50 \mathrm{~K}$ ) as a function as a function of the measured whole-sample $\mathrm{J}_{\mathrm{c}}$ (at $77 \mathrm{~K}$ ). We interpret changes in $\mathrm{J}_{\mathrm{c}}$ along the $\mathrm{X}$ axis (i.e., changes in the whole-sample $J_{c}$ ) from one sample to the next as being due to changes in connectivity between the grains. This may result from converting remnant liquid to crystalline phases, which increases the connectivity. We interpret changes in $\mathrm{J}_{\mathrm{C}}$ along the $\mathrm{Y}$ axis (i.e., changes in the local $\mathrm{J}_{\mathrm{c}}$ ) from one sample to the next as being due to changes in flux pinning resulting from changes in the oxygen content of the samples. These results show that the largest changes in connectivity occur during "post annealing” after HT1, whereas changes in flux pinning dominate during post annealing after HT2.

We believe that 2212 is a CLM in 2223. To investigate if 2212 had a deleterious effect on $\mathrm{J}_{\mathrm{c}}$, we did MOCR at temperatures ranging from 40 to $90 \mathrm{~K}$. Three local regions were selected, with low, medium, and high $\mathrm{J}_{\mathrm{c}}$ (Fig. 7). The local $\mathrm{J}_{\mathrm{c}}$ in each of these regions was plotted as a function of temperature. In all three regions, $\mathrm{J}_{\mathrm{C}}$ increased smoothly with increasing temperature. We did not see a sudden increase in $\mathrm{J}_{\mathrm{c}}$ at increasing temperature at about $\sim 80 \mathrm{~K}$, which is the $\mathrm{T}_{\mathrm{C}}$ for 2212 in the sample. This showed that 2212 did not have a significant effect on the measured $\mathrm{J}_{\mathrm{c}}$ in this region of the sample, or that MOCR was not sensitive enough to resolve local changes in $\mathrm{J}_{\mathrm{C}}$. 


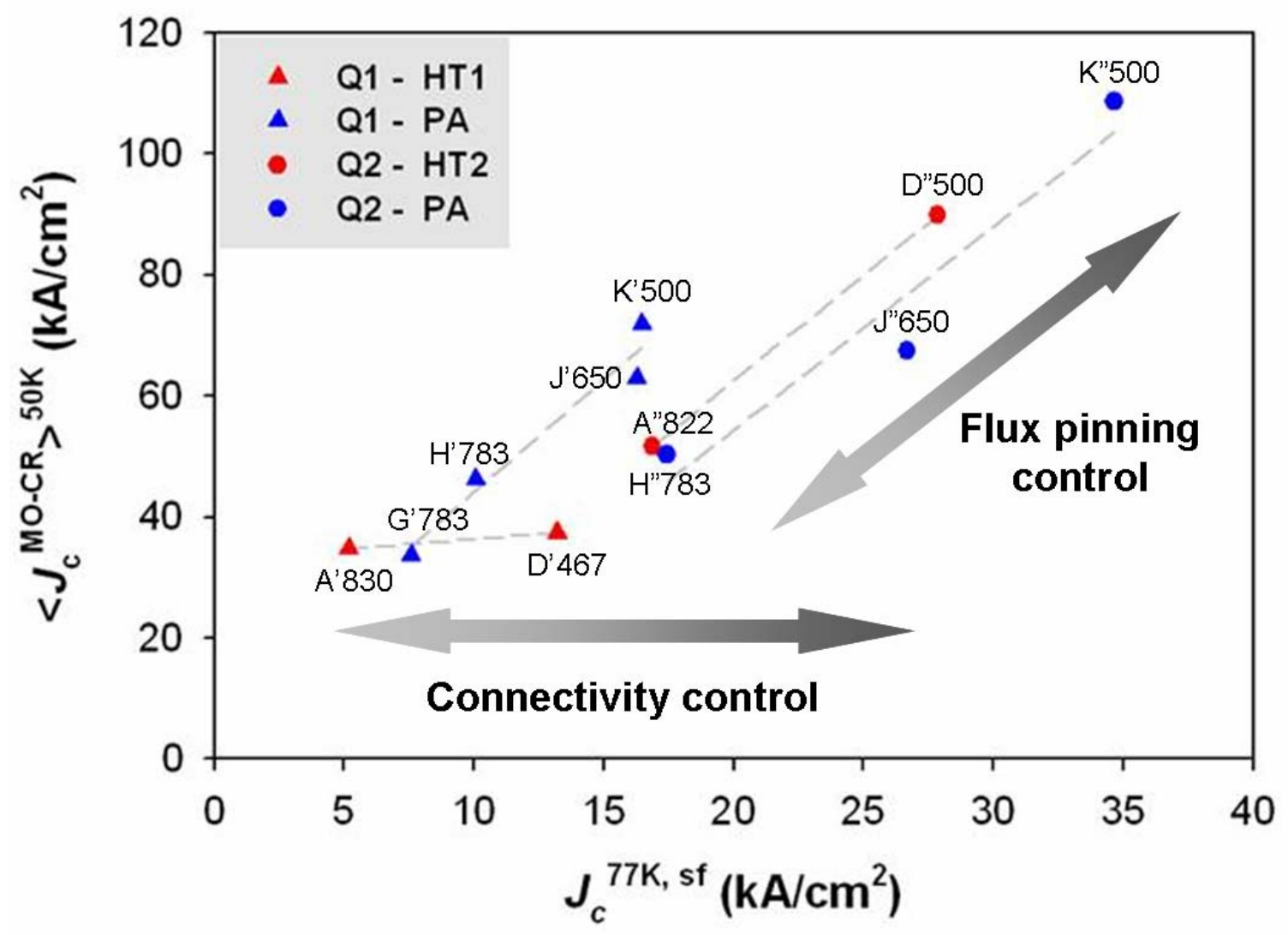

Figure 7. The average local $\mathrm{J}_{\mathrm{c}}$ measured at $50 \mathrm{~K}$ is plotted as a function of the long-range, whole-sample $\mathrm{J}_{\mathrm{C}}$ at $77 \mathrm{~K}$. Samples were quenched after the first and second heat treatments. The quenching schedule after the first heat treatment was very similar to that shown in Fig. 1. The data points have letters and numbers with the following meanings: letters indicate points in the quench sequence (' indicates a sample quenched after HT1, "indicates a sample quenched after HT 2) and the number is the quench temperature. Changes in connectivity are reflected as a horizontal displacement while changes in the flux pinning or local $J_{c}$ show up as vertical displacements. Broadly speaking HT1 influences the transport $J_{c}$ mainly by changing the connectivity, while $J_{c}$ can be raised in HT2 by optimizing the flux pinning.

Low-temperature laser scanning microscopy (LTLSM).

The low-temperature laser scanning microscope is a new instrument that we began building to measure local current dissipation in samples to determine the local positions of CLMs. Abraimov designed and began building the LTLSM. Figure 8 shows the concept of the LTLSM. The sample is in a superconducting state with a super current flowing through it that is just below $\mathrm{I}_{\mathrm{c}}$. The laser beam is rastered across the sample, locally heating the sample, thus potentially perturbing the local supercurrent flow. The voltage drop across the entire sample is recorded for each raster point and the change in voltage is used to construct a two-dimensional map which corresponds to the 2D electric field at each point on the sample. Regions with low $\mathrm{J}_{\mathrm{C}}$ due to the presence of CLMs are 

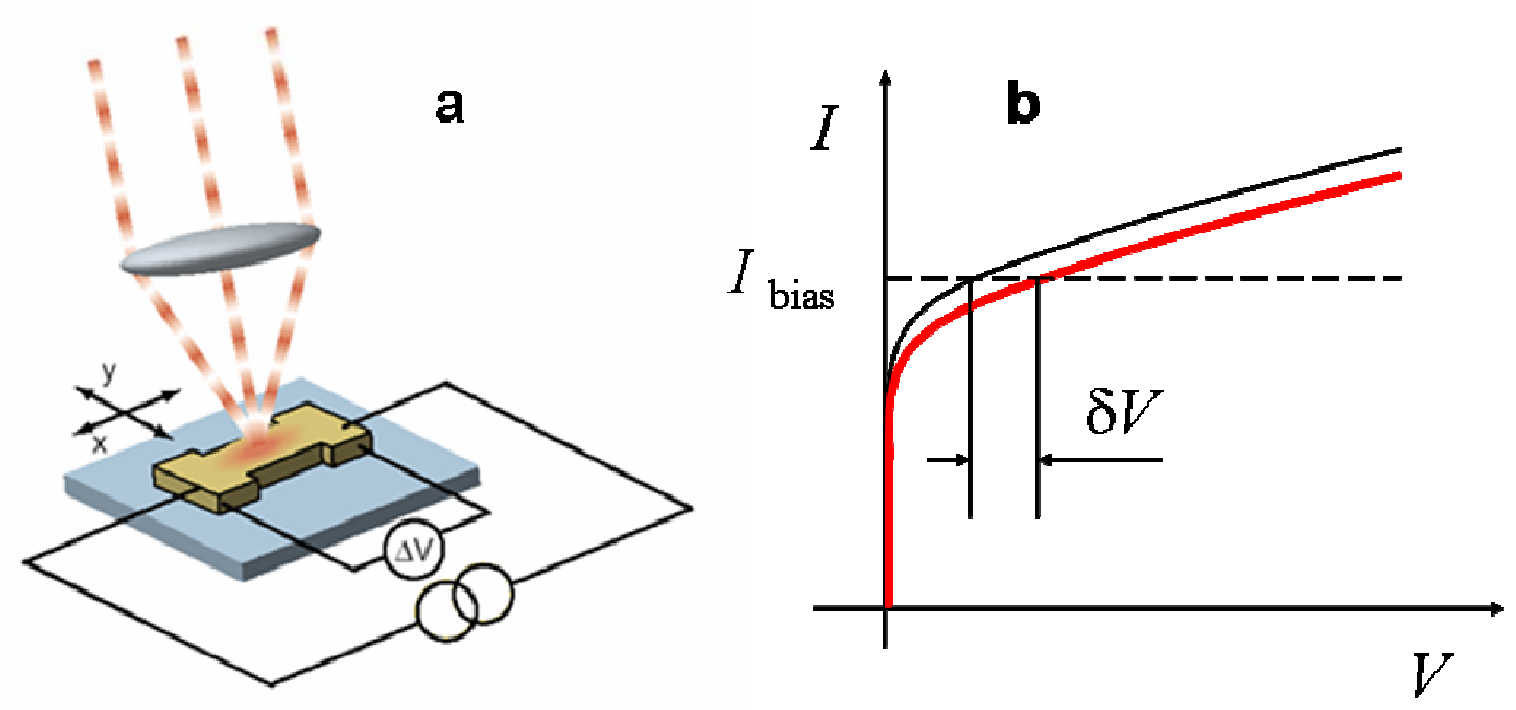

$$
\begin{gathered}
\Delta P \rightarrow \Delta T\left(x_{l}, y_{l}\right) \rightarrow \frac{\Delta \rho}{\Delta \mathrm{j}_{\mathrm{c}}} \rightarrow \delta V\left(x_{l}, y_{l}\right) \\
\Lambda_{a c}=\frac{\Lambda_{d c}}{\sqrt{\omega \tau}} \quad \delta V\left(x_{l}, y_{l}\right) \approx-\frac{\partial J_{c}}{\partial T} \frac{E\left(x_{l}, y_{l}\right)}{J_{c}} 2 n \Lambda \delta T
\end{gathered}
$$

Figure 8 (a) Schematic diagram showing the components and operation of the lowtemperature laser scanning microscope (LTLSM). (b) The change in voltage $\delta \mathrm{V}$ produced due to heating the sample in a specific spot with the laser beam.

identified. The instrument remained unfinished at the end of the contract. Subsequent tests on the completed instrument showed that its spatial resolution for current flow in coated conductors was $\sim 5 \mu \mathrm{m}$. LTLSM data on $1 \mathrm{~atm}$ and OP processed monocore wires taken by Abraimov on our BSCCO filaments at the University of Erlangen LTLSM facility are shown in Fig. 9. The dark regions in the electrical response show where high dissipation occurs in the samples. The $1 \mathrm{~atm}$ sample has significantly more dissipation than the OP processed sample. Our goal was to use the FIB to cut out strongly dissipative regions and to contrast these with high- $J_{c}$ regions so as to identify the important CLMs.

\section{PROPOSED WORK THAT WAS NOT PURSUED.}

In the processing studies, due to budget constraints, we did not carry out extensive studies investigating new compositions to try to eliminate nonsuperconducting phases and 2212. This includes not having established a freeze-dry capability to make 2223. Since the conclusion of the contract in December 2003, it has become clear that changing the overall composition of 2223 is a most promising route to raising $J_{c}$ and that it appears to be a route under considerable study in Japan. We also did not do the slide 


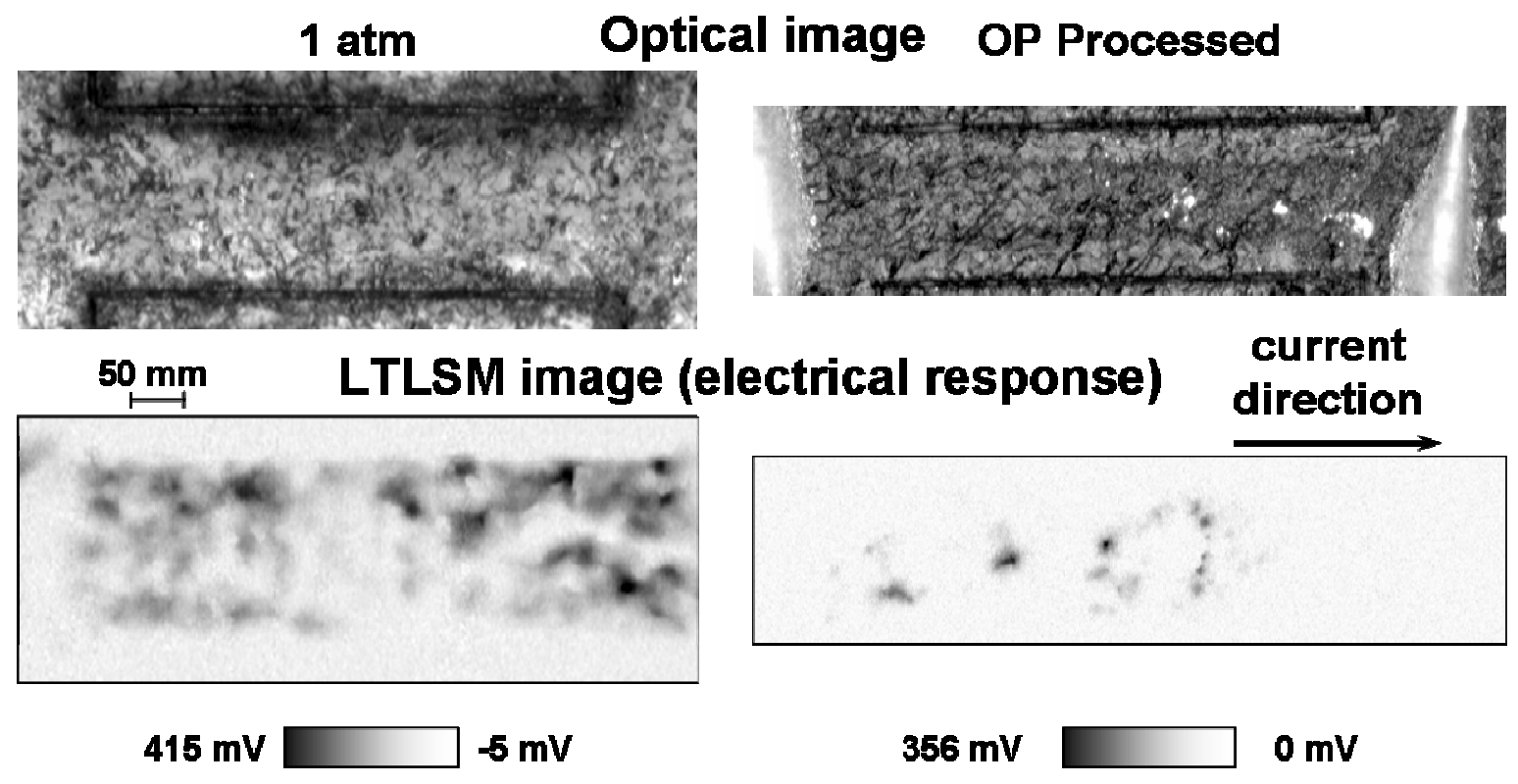

Figure 9. Optical micrographs and LTLSM (electrical response) images of a filament extracted from a $1 \mathrm{~atm}$ and an OP processed monocore tape. The dark regions in the LTLSM images are where dissipation occurs in the sample. The 1 atm sample has more dissipative regions than the OP sample.

thermal heat treatments developed by Maroni at ANL in the OP system, because of budget constraints.

In the local characterization studies, we did not use $\mu \mathrm{m}$ scale contact voltage probes to measure local I-V characteristics. Our initial studies showed it was difficult to maintain contact when cooling the sample to $77 \mathrm{~K}$, and the spatial resolution was not as good as we could achieve with MOCR and LTLSM. In addition, MOCR and LTSLM gave two-dimensional maps that showed the specific location of CLMs in the sample to within a $5 \times 5 \mathrm{~m}^{2}$ area. In contrast the contact probes only showed that a CLM existed between two voltage contacts. This located CLM within an area that was a long as the separation between the probes, which could be as small as $10 \mu \mathrm{m}$, and as wide as the 2223 filament, which could be as wide as $4000 \mu \mathrm{m}$ in a monocore wire.

\section{COLLABORATIONS}

We continued to collaborate with American Superconductor, Corp. (AMSC), Argonne National Laboratory (Vic Maroni), and Los Alamos National Laboratory (LANL) during this contract. This collaboration is called the Wire Development Group, and has been working together on 2223 wires since the early 1990s. The group met 3 times per year to discuss technical issues designed to improve the performance of AMSC's 2223 wire. A major testament to the success of our work is that we developed the highest critical current wire in the world, a record of $202 \mathrm{~A}$ in a standard cable wire. At the time that the work was discontinued, AMSC was making wire with $I_{c}$ values of 
about $150 \mathrm{~A}$ in production and about $170 \mathrm{~A}$ in short lengths with optimized post processing according to schedules shown in Fig. 2. The 202 A record was obtained with a post-processing OP step. Since 2003 Sumitomo Electric Co have industrialized OP processing, first raising their $100 \mathrm{~A}$ conductor to about $130 \mathrm{~A}$ and most recently (February 2006) to $200 \mathrm{~A}$.

\section{PUBLICATIONS}

1. J. Jiang, X.Y. Cai, J.G. Chandler, M.O. Rikel, E.E. Hellstrom, R.D. Parrella, Dingan Yu, Q. Li, M.W. Rupich, G.N. Riley Jr and D.C. Larbalestier. "Evolution of core density of Ag-clad Bi-2223 tapes during process," IEEE Trans Appl Supercond 11 (2001) 3561-4.

2. M.O. Rikel, R.K. Williams, X.Y. Cai, A.A. Polyanskii, J. Jiang, D. Wesolowski, E.E. Hellstrom, D.C. Larbalestier, K. DeMoranville and G.N. Riley Jr. "Overpressure processing Bi2223/Ag tapes," IEEE Trans Appl Supercond 11 (2001) 3026-9.

3. J. Jiang, X.Y. Cai, A.A. Polyanskii, L.A. Schwartzkopf, D.C. Larbalestier, R.D. Parrella, Q. Li, M.W. Rupich and G.N. Riley Jr. "Through-process study of factors controlling the critical current density of Ag-sheathed $(\mathrm{Bi}, \mathrm{Pb})_{2} \mathrm{Sr}_{2} \mathrm{Ca}_{2} \mathrm{Cu}_{3} \mathrm{O}_{\mathrm{x}}$ tapes," Superconductor Science and Technology 14 (2001) 548-56.

4. D. Larbalestier, A. Gurevich, D.M. Feldmann and A. Polyanskii. "High $T_{C}$ superconducting materials for electric power applications," Nature 414 (2001) 36877.

5. Y. Yuan, R.K. Williams, J. Jiang, D.C. Larbalestier, X.Y. Cai, M.O. Rikel, K.L. DeMoranville, Y. Huang, Q. Li, E. Thompson, G.N. Riley Jr. and E.E. Hellstrom. "Overpressure processing of Ag-sheathed $(\mathrm{Bi}, \mathrm{Pb})_{2} \mathrm{Sr}_{2} \mathrm{Ca}_{2} \mathrm{Cu}_{3} \mathrm{O}_{\mathrm{x}}$ tapes," Physica $\mathrm{C}$ 372-376 (2002) 883-6.

6. J. Jiang, X.Y. Cai, J.G. Chandler, S. Patnaik, A.A. Polyanskii, Y. Yuan, E.E. Hellstrom and D.C. Larbalestier. "Critical current limiting factors in post annealed $(\mathrm{Bi}, \mathrm{Pb})_{2} \mathrm{Sr}_{2} \mathrm{Ca}_{2} \mathrm{Cu}_{3} \mathrm{O}_{\mathrm{x}}$ tapes," IEEE Trans Appl Supercond 13 (2003) 3018-21.

7. S. Patnaik, D.M. Feldmann, A.A. Polyanskii, Y. Yuan, J. Jiang, X.Y. Cai, E.E. Hellstrom, D.C. Larbalestier and Y. Huang. "Local measurement of current density by magneto-optical current reconstruction in normally and overpressure processed Bi-2223 tapes," IEEE Trans Appl Supercond 13 (2003) 2930-3.

8. Y. Yuan, J. Jiang, X.Y. Cai, S. Patnaik, A.A. Polyanskii, E.E. Hellstrom, D.C. Larbalestier, R.K. Williams and Y. Huang. "Microstructure and $\mathrm{J}_{\mathrm{c}}$ improvements in overpressure processed Ag-sheathed Bi-2223 tapes," IEEE Trans Appl Supercond 13 (2003) 2921-5. 
9. J.G. Chandler, J. Jiang, X.Y. Cai, L.A. Schwartzkopf and D.C. Larbalestier. "Irreversibility fields of Bi-2223 at 30-77 K," IEEE Trans Appl Supercond 13 (2003) 2945-8.

10. O. van der Meer, X. Cai, J. Jiang, R.D. Parella, Y.B. Huang, D.C. Larbalestier, B. ten Haken and H.H.J. ten Kate. "Characterization of individual filaments extracted from a Bi-2223/Ag tape," IEEE Trans Appl Supercond 13 (2003) 2926-9.

11. Y.B. Huang, X.Y. Cai, T. Holesinger, V.A. Maroni, D. Yu, R. Parrella, M. Rupich, E. Hellstrom, M. Teplitsky, K. Venkataraman, A. Otto and D. Larbalestier. "Improving the critical current density in Bi-2223 wires via a reduction of the secondary phase content," IEEE Trans Appl Supercond 13 (2003) 3038-41.

12. Y. Yuan, X. Cai, J. Jiang, Y. Huang, R. Parrella, D. Larbalestier and E. Hellstrom. "Overpressure processing and characterization of silver sheathed $(\mathrm{Bi}, \mathrm{Pb})_{2} \mathrm{Sr}_{2} \mathrm{Ca}_{2} \mathrm{Cu}_{3} \mathrm{O}_{\mathrm{x}}$ composite conductors," Mater Res Soc Symp Proc EXS-3 (2004) 145-7.

13. Y. Yuan, J. Jiang, X.Y. Cai, D.C. Larbalestier, E.E. Hellstrom, Y. Huang and R. Parrella. "Significantly enhanced critical current density in Ag-sheathed $(\mathrm{Bi}, \mathrm{Pb})_{2} \mathrm{Sr}_{2} \mathrm{Ca}_{2} \mathrm{Cu}_{3} \mathrm{O}_{\mathrm{x}}$ composite conductors prepared by overpressure processing in final heat treatment," Appl Phys Lett 84 (2004) 2127-9.

14. A. Polyanskii, V. Beilin, M. Feldmann, M. Roth, E. Hellstrom and D. Larbalestier. "Magneto-optical investigation of superconducting materials," NATO Science Series, II: Mathematics, Physics and Chemistry 142 (2004) 19-28.

15. R.M. Scanlan, A.P. Malozemoff and D.C. Larbalestier. "Superconducting materials for large scale applications," Proc IEEE 92 (2004) 1639-54.

16. S. Liao, E.E. Hellstrom and D.C. Larbalestier. "Correlation of local magneto-optical measurements of critical current density to microstructure in Bi-Sr-Ca-Cu-O superconducting tapes," Mater Res Soc Symp Proc EXS-3 (2004) 101-3.

17. Y.B. Huang, M. Aviza, B. Carter, K. Demoranville, C. King, S. Fleshler, A. Otto, E. Podtburg, J. Schreiber, A.P. Malozemoff, X.Y. Cai, E. Hellstrom, D. Larbalestier, T. Holesinger and V.A. Maroni. "Enhanced performance of first generation HTS wire," Mater Res Soc Symp Proc EXS-3 (2004) 141-3.

18. T.G. Holesinger, J.A. Kennison, S. Liao, Y. Yuan, J. Jiang, X.Y. Cai, E.E. Hellstrom, D.C. Larbalestier, R.M. Baurceanu, V.A. Maroni and Y. Huang. "A shell model for the filament structure of Bi-2223 conductors," IEEE Trans Appl Supercond 15 (2005) 2514-17.

19. J. Jiang, X.Y. Cai, Y. Yuan, A.A. Polyanskii, E.E. Hellstrom, D.C. Larbalestier, V.A. Maroni, T.G. Holesinger and Y. Huang. "Long term anneal study and composition variation for reducing residual $\mathrm{Bi}_{2} \mathrm{Sr}_{2} \mathrm{CaCu}_{2} \mathrm{Ox}(2212)$ in $(\mathrm{Bi}, \mathrm{Pb})_{2} \mathrm{Sr}_{2} \mathrm{Ca}_{2} \mathrm{Cu}_{3} \mathrm{O}_{\mathrm{x}}$ (2223) wires," IEEE Trans Appl Supercond 15 (2005) 2526-9. 
20. Y. Yuan, X.Y. Cai, J. Jiang, Y. Huang, D.C. Larbalestier and E.E. Hellstrom. "The origin of the $\mathrm{Pb}$-rich $\mathrm{Bi}_{3} \mathrm{Sr}_{2} \mathrm{Ca}_{2} \mathrm{CuO}_{\mathrm{x}}$ (3221) phase during post annealing of $(\mathrm{Bi}, \mathrm{Pb})_{2} \mathrm{Sr}_{2} \mathrm{Ca}_{2} \mathrm{Cu}_{3} \mathrm{O}_{\mathrm{x}} \mathrm{Ag}$ composite conductors," IEEE Trans Appl Supercond 15 (2005) 2530-3.

\section{PATENTS}

1. Q. Li, E.D. Thompson, J.N. Riley, Jr., E.E. Hellstrom, D.C. Larbalestier, K.L DeMoranville, J.A. Parrell, J.L Reeves, "Simultaneous constraint and phase conversion processing of oxide superconductors,” \#6,555,503, Apr. 29, 2003 